\title{
Experimental Applications of the Nuclear Equation-of-State to Neutron Star Dynamics
}

Michael A. Famiano*广

Western Michigan University

E-mail: michael.famiano@wmich.edu

\section{William G. Lynch}

National Superconducting Cyclotron Laboratory

E-mail: Iynch@nscl.msu.edu

\section{Betty Tsang}

National Superconducting Cyclotron Laboratory

E-mail: tsang@nscl.msu.edu

The characterization of the density dependence of the nuclear equation-of-state (EOS) remains a longstanding problem in nuclear astrophysics. While observations of neutron star masses have placed astronomical lower limits on the asymmetry term of the EOS, experimental constraints remain open. Recent improvements in experiment, data interpretation, and theoretical predictions have greatly reduced the constraints on what is known concerning this dependence. Future work must focus on studying the nuclear EOS at higher-density $\left(\rho>\rho_{o}\right)$. Recent heavy-ion beam experimentation will be described, and the theoretical interpretation will be presented. Of particular importance are plans to increase the density of the explored region of the nuclear EOS. The applications of experimental results from ${ }^{40,48} \mathrm{Ca}+{ }^{112,124} \mathrm{Sn}$ reactions at $140 \mathrm{MeV} / \mathrm{A}$ will also be covered. These reactions were studied to constrain the in-medium nucleon masses, which are a considerable unknown in model predictions of the nuclear EOS.

11th Symposium on Nuclei in the Cosmos, NIC XI

July 19-23, 2010

Heidelberg, Germany

\footnotetext{
* Speaker.

${ }^{\dagger}$ Work Supported by NSF Grant \#PHY-0757257
} 


\section{Introduction}

Studies have placed significant constraints upon the equation-of-state (EOS) for isospin symmetric nuclear matter. The dependence of the EOS on the isospin asymmetry $\delta=\left(\rho_{n}-\rho_{p}\right) /\left(\rho_{n}+\right.$ $\left.\rho_{p}\right)$, however, remains largely unconstrained; estimates of the uncertainty due to this term suggest that it may be the dominant contribution to the uncertainty in the EOS of neutron matter. Not surprisingly, the asymmetry term governs many macroscopic properties of neutron stars, such as their radii, their moments of inertia, their maximum masses, how they are formed and cool, [1] and the possibility that exotic matter may occur in the neutron star core. The radii and moments of inertia are particularly sensitive to the density dependence of the asymmetry term at normal and sub-normal density. However, the uncertainty in the pressure due to the nuclear asymmetry term can be larger than many estimates for the total pressure of the system. Measurements at the NSCL have recently provided information about the density dependence of the asymmetry term. [2, 3, 4, 5] Observables which have been measured and which are predicted to be sensitive to the isospin dependence of the EOS include isospin diffusion, neutron-proton emission ratios, [3] and most recently charged-particle correlations.

\section{Experimental Work}

Work has been completed on NSCL experiment \#07018 and \#05049 to explore the isospin dependence of the in-medium nuclear cross-sections. In this experiment, measurements of the relative neutron and proton flow observables were made to better understand and constrain the in-medium nucleon cross-sections, which are an unknown parameter in transport calculations. [6] For an effective experiment, a fairly complicated setup was done which included neutron detectors with a large angular range, the Large Area Neutron Array (LANA)[7] charged particle telescopes capable of a similar angular range, and a sufficient energy range to provide emission spectra extracted from the mid-rapidity region in the described reactions, and way of measuring the reaction impact parameter via charged particle multiplicity. These devices already exist in the form of the large liquid scintillator neu-

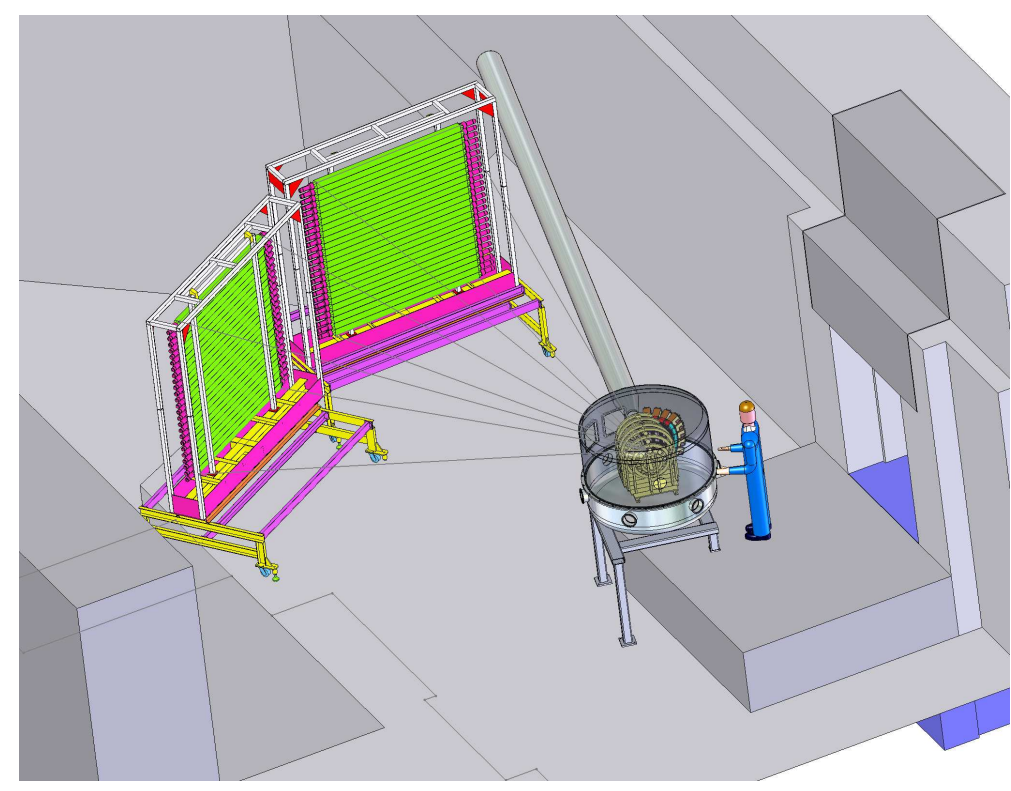

Figure 1: Technical drawing of the NSCL S2 reactions area showing the neutron TOF walls and scattering chamber. tron walls at the NSCL, [7] elements of the LASSA array and the MiniBall array. [8] Additionally, a 
simple position sensitive trigger detector array was constructed for timing and acquisition triggering.

The experimental setup is shown in Figure 1. Neutron walls were placed a distance of about 6-7 $\mathrm{m}$ from the target with an angular coverage in the lab of $8^{0}$ to $60^{\circ}$, providing a center-of-mass coverage of $70^{\circ}$ to $110^{\circ}$ for the EOS experiment, as well as a large angular coverage at forward angles for the NN cross-section measurement. A similar coverage was provided by six chargedparticle telescopes. The S2 vault is large enough to accommodate the neutron walls north of the target area.

Impact parameter was determined via charged-particle multiplicity in the MiniBall[8], which was complimented by a granular plastic scintillator start detector. The Microball provides over 97\% coverage over backward angles, as well as particle identification. The combined information from the charged-particle telescopes, the start detector information, and the MiniBall provided a good measure of the impact parameter and reaction plane needed for proper event characterization.

This experiment utilized ${ }^{(40,48)} \mathrm{Ca}+{ }^{(112,124)} \mathrm{Sn}$ reactions at $140 \mathrm{MeV} / \mathrm{u}$. Neutron and chargedparticle kinematics were measured. Analysis is currently underway, and preliminary results are being produced. As an example, neutron energy spectra are shown in Figure 2 for the ${ }^{48} \mathrm{Ca}+{ }^{124} \mathrm{Sn}$ reaction, showing neutron energies well above $200 \mathrm{MeV}$. this is important as sensitivity to in-medium effective masses is greatest at highest particle rapidities. It is particularly interesting to note that the neutron wall also makes an excellent chargedparticle detector. Charged particles are detected via their TOF-charge deposit relationship. This may be extremely useful as we may very well be able to extract neutron-proton correlation functions, which may be a good indicator of the stiff-

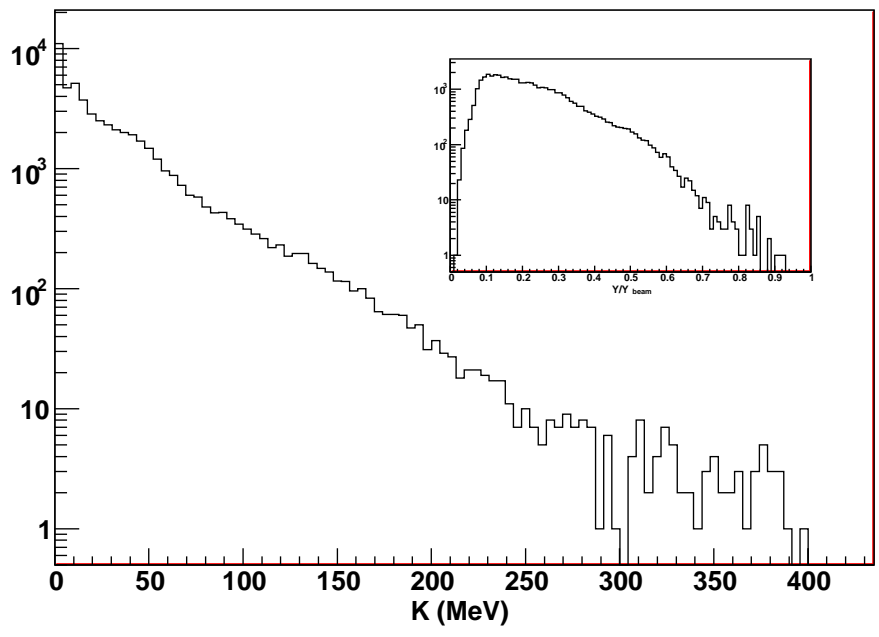

Figure 2: Neutron energy spectra from the ${ }^{48} \mathrm{Ca}+{ }^{124} \mathrm{Sn}$ reaction in NSCL experiment \#05049. Note that the neutron energies well above $200 \mathrm{MeV}$ are achievable, meaning that high particle rapidity should be observed. The inset shows the rapidity distribution of the produced neutrons in the lab frame (relative to the beam), indicating production at relative rapidities above 0.7 where the sensitivity to the effective masses is greatest. Only a fraction of the data is shown. ness of the asymmetry term of the nuclear EOS.[9] This effect is explored as the momentum dependence (interaction term) results in a reduction off the in-medium effective masses, which in turn results in a reduction of the in-medium cross-sections. A reduction of the in-medium cross-sections will alter the asymmetry of forward-emitted fragments as projectile decay fragments must undergo transport through the excited medium. Likewise, a larger neutron attraction resulting from a stiffer low-density EOS will result in more correlated neutron emission. 


\section{Computational Work}

The experiment is supplemented by theoretical investigations of the density dependence of the asymmetry energy in the nuclear equation-of-state (EOS). Two primary investigations have been completed using transport calculations to constrain predictions of the asymmetry term of the nuclear EOS with experimental observations. Prior calculations [10, 11] have been shown to be somewhat inconsistent with each other and with experimental results. [3] This inconsistentcy stems from the fact that the effects of clusters (both formation and decay) are treated in an inconsistent manner. Recent calculations have attempted to reduce this uncertainty by investigating preequilibrium emissions from the same reactions investigated in prior experiments[3] using both particle transport and quantum molecular dynamics (QMD) models. [12] These calculations have been adapted to both clustering and secondary decay codes.

In one study, the emissions of neutrons, protons and bound clusters from central ${ }^{124} \mathrm{Sn}+{ }^{124} \mathrm{Sn}$ and ${ }^{112} \mathrm{Sn}+{ }^{112} \mathrm{Sn}$ collisions have been simulated using the Improved Quantum Molecular Dynamics model for two different densitydependent symmetry-energy functions. The calculated neutron-proton spectral double ratios for these two systems have been shown to be sensitive to the density dependence of the symmetry energy, consistent with previous work. In this investigation, the double ratios, defined to be the ratio of total neutron-proton emission ratios for each system:

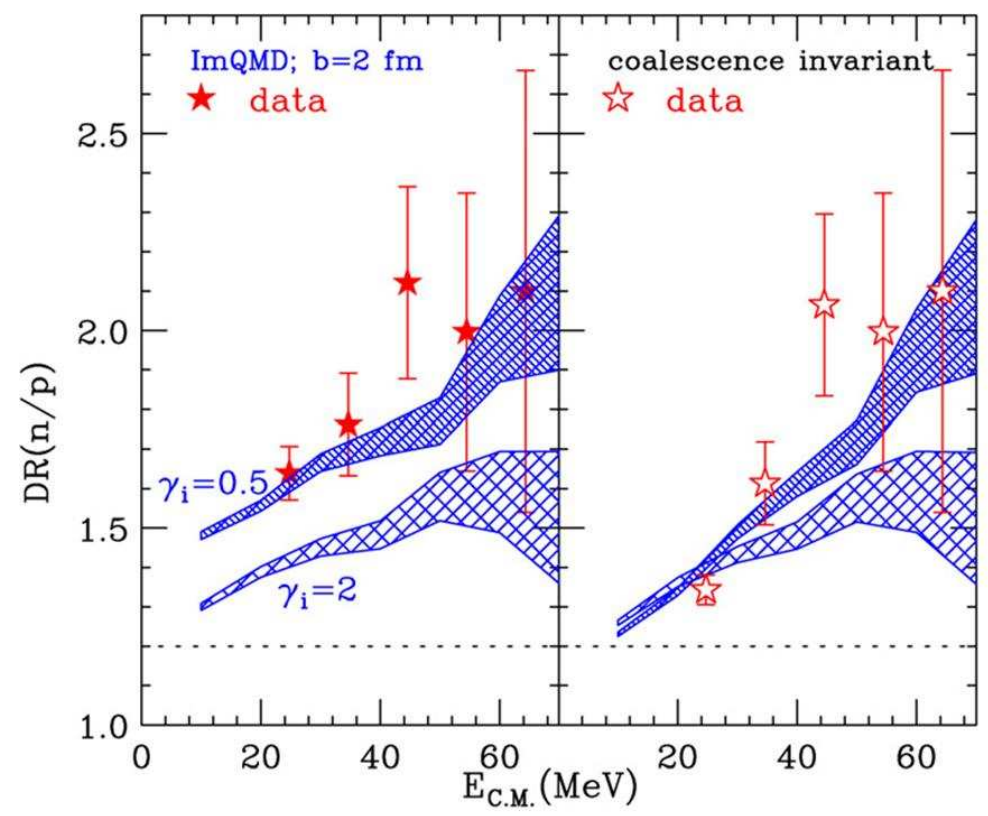

Figure 3: The free neutron-proton double-ratio (left panel), and the coalescence- invariant neutronproton double-ratios (right panel) plotted as a function of kinetic energy of the nucleons. The shaded regions represent calculated results from the ImQMD simulations at $b=2 \mathrm{fm}$. Experimental data is shown.[3]

$$
D R_{(n / p)} \equiv\left(\frac{\frac{d Y_{n}}{d E}}{\frac{d Y_{p}}{d E}}\right)_{124} /\left(\frac{\frac{d Y_{n}}{d E}}{\frac{d Y_{p}}{d E}}\right)_{112}
$$

However, cluster emission increases the double ratios in the low energy region relative to values calculated in a "coalescence-invariant" approach, that is, an analysis in which all nucleons are counted, both free and bound in clusters. To circumvent uncertainties in cluster production and secondary decays, it is important to have more accurate measurements of the neutron-proton ratios at higher energies in the center of mass system, where the influence of such effects is reduced. The details of the calculation are described in Zhang et al. (2008).[12] 
While previous calculations using the BUU model[11] have shown a lack of sensitivity of the neutron-proton emission for a momentum-dependent mean-field potential (and hence, a disagreement with theory), recent calculations incorporting this coalescent-invariant approach have shown a reasonable agreement with experimental results. Models using the ImQMD[13] calculation are shown in Figure 3 for free nucleons and all nucleons (coalescent invariant). In this figure, the symmetry energy of the EOS is parametrized as $S(\rho) \propto \rho^{\gamma}$. While the agreement is good, it can also be seen that the isotopic emission ratios at highter energies have large uncertainties due to low statistics. Reducing this uncertainty is one area of concentration in the upcoming years.

In addition to these recent calculations, a chi-square analysis has been applied to recent theoretical results to constrain parameters of the asymmetry term in calculations. These analyses concentrate on constraining results from isospin diffusion as well as from isotopic ratios in a consistent manner. Calculations have recently been completed, and results are currently in press.

\section{References}

[1] J.M. Lattimer \& J.M. Prakash, Phys. Rep.442 (2007) 109.

[2] V. Henzl,D. Henzlova,M.A. Famiano,W.G. Lynch, M.B. Tsang,M. Kilburn,J. Lee,A.M. Rogers,G. Verde, \& M. Youngs, The $10^{\text {th }}$ International Symposium on Nucleus-Nucleus Collsisions, August 16 21, 2009, Beijing, China.

[3] M.A. Famiano, T. Liu, T., W.G. Lynch, A.M. Rogers, M.B. Tsang, M.S. Wallace, R.J. Charity, S. Komarov, D.G. Sarantites, \& L.G., Sobotka, Phys. Rev. Lett. 97 (2006) 052701.

[4] T.X. Liu, W.G. Lynch, M.B, Tsang, R. Shomin, W.P. Tan, G. Verde, A.agner, H.F. Xi, H.S. Xu, B. Davin, Y. Larochelle, R.T. de Souza, R.J. Charity, \& L.G. Sobotka, Phys. Rev. C. 76 (2007) 034603.

[5] M.B. Tsang, Y. Zhang, P. Danielewicz, M. Famiano, Z. Li, W.G. Lynch, \& A.W. \& Steiner, Phys. Rev. Lett. 1022009122701.

[6] L.-W. Chen, C.M. Ko, \& B.-A. Li, Phys. Rev. C 69 (2004) 054606.

[7] P.D. Zecher, et al., Nucl. Instr. \& Meth. in Phys. Res. A 401 (1997) 329.

[8] R.T. deSouza, et al., Nucl. Instr. \& Meth. in Phys. Res. A 295 (1990) 109.

[9] L. -W. Chen, C.M. Ko, \& B.-A. Li, Phys. Rev. Lett. 90 (2003) 162701.

[10] B. -A. Li, C. Ko \& Z. Ren, Phys. Rev. Lett. 78 (1997) 1644.

[11] B.-A. Li, L. W. Chen, G.C. Yong, \& W. Quo, Phys. Lett. B 634 (2006) 378.

[12] Y. Zhang, P. Danielewicz, M. Famiano, Z. Li, W. G. Lynch, \& M.B. Tsang, Phys. Lett. B 664 (2008) 145.

[13] M.B. Tsang, Y. Zhang, P. Danielewicz, M. Famiano, et al., Phys. Rev. Lett. 102 (2009) 122701. 